\title{
468879 - POSTOPERATIVE TROPONIN I LEVELS AND SIDE EFFECTS FOLLOWING METHYLPREDNISOLONE ADMINISTRATION IN CHILDREN UNDERGOING CARDIOPULMONARY BYPASS
}

\author{
Sylvie Aucoin, $\mathrm{MD}^{1}$, Arnim Vlatten, $\mathrm{MD}^{1}$, Sharon Litz, $\mathrm{MD}^{2}$, Chris Soder, $\mathrm{MD}^{2}$, \\ Brian MacManus, MD $^{2}$ \\ 1. Anesthesia, Dalhousie University, Halifax, NS, Canada \\ 2. Pediatric Anesthesia, Dalhousie University, Halifax, NS, Canada
}

Introduction: It has been suggested that steroid treatment prior to pediatric cardiopulmonary bypass (CPB) halts the systemic inflammatory response, possibly reducing myocardial damage and improving patient outcome. The effectiveness of this common practice remains unclear. We examined whether methylprednisolone (MP) administration prior to CPB affected postoperative troponin I (cTn1) levels, a known specific marker for myocardial cell damage.

Methods: Following ethics committee approval in a pediatric university hospital, a retrospective chart review was carried out in children undergoing open heart surgery between April 2004 and August 2005. A total of 57 children were included. Exclusion criteria were: fever $\left(>38.5^{\circ} \mathrm{C}\right)$, white cell count $>12,000$ cells $/ \mathrm{mm} 3$ and preoperative use of steroids or NSAIDS. Following induction of anesthesia, the anesthetist administered as their choice either methylprednisolone $20 \mathrm{mg} / \mathrm{kg}$ (group MP, N=25), or no steroid (group $\mathrm{CON}, \mathrm{N}=32$ ). Troponin I levels were recorded at time point 0 (immediately on arrival to PICU) and 24 hours postoperatively as routine care. Secondary outcomes were: serum glucose levels, perioperative catecholamine requirements, and postoperative morbidity and mortality.

Results: There was no significant difference in troponin I levels between the two groups at both time points (see graph). There was also no significant difference in glucose and catecholamine administration, length of PICU stay, wound infection or perioperative mortality. However, there was a statistically significant difference $(p<0.001)$ in serum glucose levels (mmol/l) at time point 0 - CON group $9.6(6.2 ; 12.6)$ and MP group 13.0 (9.7; 18.2) (Median, 25\% and 75\% confidence interval). At 24h, serum glucose levels were CON 5.5 (4.7; 6.8) and MP 5.5 (4.8; 6.5).

Discussion: We were unable to demonstrate a significant decrease in troponin I levels at both time points. There was a large intra-group variability in troponin I levels possibly due to surgical technique and CPB characteristics. Subjects receiving MP did, however, have significantly higher serum glucose levels on arrival to PICU. Given the controversy regarding the benefit of perioperative methylprednisolone administration(1), inconsistent use among pediatric anesthesiologists (2) and clinically relevant side effects (3), further studies are required to define the role of steroids in pediatric cardiac anesthesia.

References: 1. Chaney MA, Durazo-Arvizu R, et al. J Thorac Cardiovasc Surg 2001, 121:561-9 2. Checchia PA, Bronicki RA, et al. Pediatric Crit care Med 2005, 6:441-4 3. Yates AR, Dyke PC et al. Pediatr Crit Care Med. 2006, 7:351-5 
cTnl levet at 0 and 24 hours

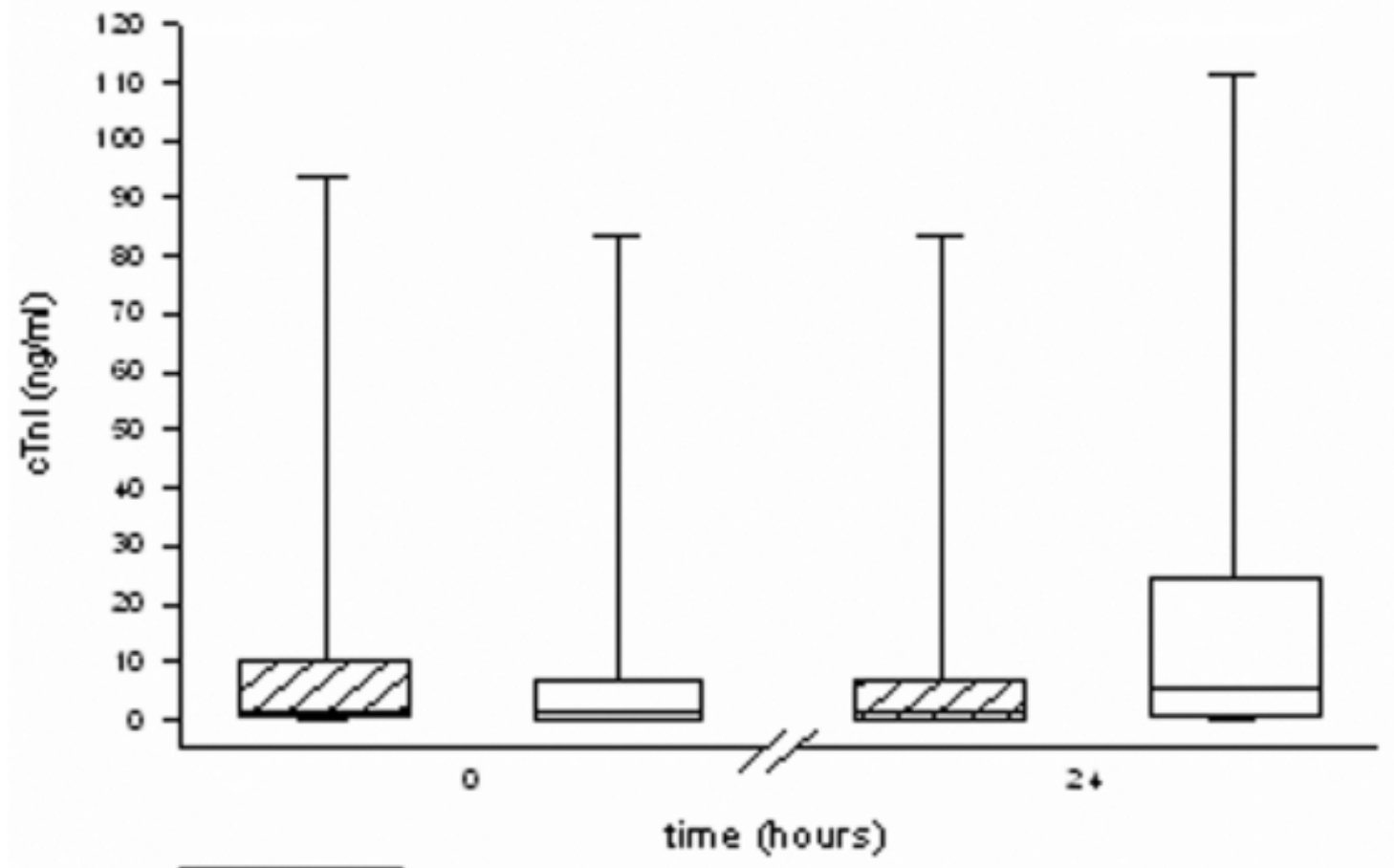

\begin{tabular}{l}
\hline C con \\
$\square$ up \\
\hline
\end{tabular} 